\title{
Probabilistic models and inference algorithms for neuronal decoding of UP and DOWN states Zhe Chen*1,2, Sujith Vijayan ${ }^{2,3}$, Riccardo Barbieri ${ }^{1}$, Matthew A Wilson ${ }^{2}$ and Emery N Brown ${ }^{1,2,4}$
}

\author{
Address: ${ }^{1}$ Neuroscience Statistics Research Lab, Massachusetts General Hospital, Boston, MA 02114, USA, ${ }^{2}$ Department of Brain and Cognitive \\ Sciences, MIT, Cambridge, MA 02139, USA, ${ }^{3}$ Program in Neuroscience, Harvard University, Cambridge, MA 02139, USA and ${ }^{4}$ Harvard-MIT \\ Division of Health Sciences and Technology, Cambridge, MA 02139, USA \\ Email: Zhe Chen* - zhechen@ neurostat.mgh.harvard.edu \\ * Corresponding author
}

from Seventeenth Annual Computational Neuroscience Meeting: CNS*2008

Portland, OR, USA. 19-24 July 2008

Published: II July 2008

BMC Neuroscience 2008, 9(SuppI I):P32 doi:I0.I 186/I47I-2202-9-SI-P32

This abstract is available from: http://www.biomedcentral.com/I47I-2202/9/SI/P32

(c) 2008 Chen et al; licensee BioMed Central Ltd.

\section{Background}

In the neuroscience literature, periods during which populations of neurons are either simultaneously depolarized or hyperpolarized are often classified as "UP" and "DOWN" states, respectively [1]. No particular attention has been devoted to accurately characterize the transition between these two states within a statistical framework [2]. We propose two (semi-) Markov probabilistic models, in both discrete- and continuous-time domains, aiming to infer a discrete two-state (UP vs. DOWN) latent process based on multi-unit spike train observations. The simultaneously recorded spike trains, treated as stochastic point processes, are modulated by the discrete hidden state and the firing history of ensemble neurons. To jointly estimate the hidden state and the unknown parameters of the probabilistic models, we develop statistical inference algorithms within the maximum likelihood estimation framework.

\section{Data}

Simultaneous recordings of electroencephalograph (EEG) and multi-unit spike trains are collected from the primary somatosensory cortex and hippocampus of behaving rats during (RUN) behavior and sleep. For the purpose of the UP/DOWN state analysis, only data from slow wave sleep (SWS) sessions are analyzed.

\section{Methods}

The UP/DOWN states are treated as a latent two-state Markov or semi-Markov process. The multi-unit spike trains are modeled as state-dependent Markov-modulated Cox processes. Given the observed spike trains, the missing data estimation problem can be tackled efficiently by maximum likelihood estimation. We develop a discretetime two-state hidden Markov model (HMM) and the associated expectation maximization (EM) algorithm [3] for estimating the UP and DOWN states. Using estimates obtained from this analysis, we further develop a continuous-time semi-Markov model for inferring the sojourn time probability distributions that characterize the transition between the two states. This inference is tackled by a Monte Carlo EM (MCEM) algorithm, including implementation of a reversible jump Markov chain Monte Carlo (RJMCMC) sampling procedure [4].

\section{Discussion}

The discrete-time HMM provides a fast and reasonable estimate of the hidden states; however, it is prone to converge to a local solution that is far away from the optimum estimate. The continuous-time semi-Markov model uses the RJMCMC to incrementally estimate where and how many state transitions occur during the inference procedure, which yields more reliable estimation results (as confirmed by analysis of synthetic data). Using different methods, we compared the EEG-triggered average based on the estimated UP-state start and end time. 
Results suggested that the EEG-triggered (K-complex) average from the continuous-time model produced a more accurate onset of the UP state.

\section{Acknowledgements}

The research was supported by NIH/NHLBI grant R0I-HL084502 for Z. Chen and R. Barbieri, NIH/NIDA grant ROI-DAOI5644 to E. N. Brown and M. A. Wilson. S. Vijayan was supported by NIH/NRSA grant T32 HL0790I.

\section{References}

I. Sirota A, Csicsvari J, Buhl D, Buzsaki G: Communication between neocortex and hippocampus during sleep in rodents. Proc Nat Acad Sci USA 2003, 100:2065-2069.

2. Ji D, Wilson MA: Coordinated memory replay in the visual cortex and hippocampus during sleep. Nature Neurosci 2007, I0(I): 100-107.

3. Smith AC, Brown EN: Estimating a state-space model from point process observations. Neural Computat 2003, I5:965-991.

4. Ball F, Cai $Y$, Kadane JB, O'Hagan A: Bayesian inference for ionchannel gating mechanisms directly from single-channel recordings, using Markov chain Monte Carlo. Proc. Royal. Soc. Lond. A 1999, 455:2879-2932.

Publish with Bio Med Central and every scientist can read your work free of charge

"BioMed Central will be the most significant development for disseminating the results of biomedical research in our lifetime. "

Sir Paul Nurse, Cancer Research UK

Your research papers will be:

- available free of charge to the entire biomedical community

- peer reviewed and published immediately upon acceptance

- cited in PubMed and archived on PubMed Central

- yours - you keep the copyright

Submit your manuscript here:

http://www.biomedcentral.com/info/publishing_adv.asp 